\title{
IN SITU ACCELERATOR ANALYSES OF PALLADIUM COMPLEX UNDER DEUTERIUM PERMEATION
}

\author{
A. KITAMURA, R. NISHIO, H. IWAI, R. SATOH, A. TANIIKE AND Y. FURUYAMA \\ Department of Environmental Energy Science, \\ Graduate School of Science and Technology, Kobe University \\ 5-1-1 Fukaeminami-machi, Higashinada-ku, Kobe 6580022, Japan
}

\begin{abstract}
Preliminary results of experiments on $\mathrm{D}_{2}$ gas permeation using a system [vacuum/CaO/Sr/PdD $/ \mathrm{D}_{2}$ ] have shown some evidence of nuclear transmutation from $\mathrm{Sr}$ to Mo. The system is a little simpler than that used by Iwamura et al., and has a reversed gas flow direction. The diagnostic method used to identify the elements was conventional XPS, giving the areal densities of $4.2 \times 10^{14} \mathrm{~cm}^{-2}(\mathrm{Sr})$ and $3.3 \times 10^{14} \mathrm{~cm}^{-2}(\mathrm{Mo})$. Extended analytical methods are now being prepared, including in situ and simultaneous PIXE, RBS and NRA/ERD analyses for areal densities of transmutation elements and deuterium distribution.
\end{abstract}

\section{Introduction}

It has been claimed $[1,2]$ that forced permeation of deuterium through $\mathrm{Pd} /(\mathrm{CaO}+\mathrm{Pd}) / \mathrm{Pd}$ samples doped with some element $\mathrm{X}$ induced nuclear transmutations from $\mathrm{X}$ to $\mathrm{X}$ ', where (X, X') being $\left({ }^{133} \mathrm{Cs},{ }^{141} \mathrm{Pr}\right),\left({ }^{88} \mathrm{Sr},{ }^{96} \mathrm{Mo}\right),\left({ }^{138} \mathrm{Ba},{ }^{150} \mathrm{Sm}\right)$ and $\left({ }^{137} \mathrm{Ba},{ }^{149} \mathrm{Sm}\right)$. The main diagnostic methods in these studies were X-ray photoelectron spectroscopy (XPS), timeof-flight secondary-ion mass spectroscopy (TOF-SIMS) and X-ray fluorescence (XRF). To confirm and investigate the phenomena, it is essential that the same results be obtained in different laboratories with different analytical methods.

We have constructed an experimental system, with which accelerator analyses of the samples including particle induced X-ray emission (PIXE), elastic recoil detection analysis (ERDA), nuclear reaction analysis (NRA) and Rutherford backscattering spectroscopy (RBS) can be made in situ and simultaneously with gas permeation through the samples.

In the present work, we use a sample similar to, but somewhat different from those used in refs. [1] and [2]: vacuum $/ \mathrm{CaO} / \mathrm{Sr} / \mathrm{PdD}_{\mathrm{x}} / \mathrm{D}_{2}$. Here we report preliminary experimental results on the transmutation diagnosed by conventional XPS method. Results from the in situ analyses will be published later, elsewhere.

\section{Permeation-in situ Analysis System and Sample Preparation}

The experimental setup is shown in Figure 1. The multilayered sample is placed at the center of the vacuum chamber. The sample surface can be diagnosed in situ with probe beam ions to emit characteristic X-rays which are analyzed either with a CdTe detector or a Si-PIN-type X-ray detector positioned at 150 degree relative to the probe beam direction. Additional solid-state charged-particle detectors are provided for RBS, ERDA and/or NRA characterization of the sample. 


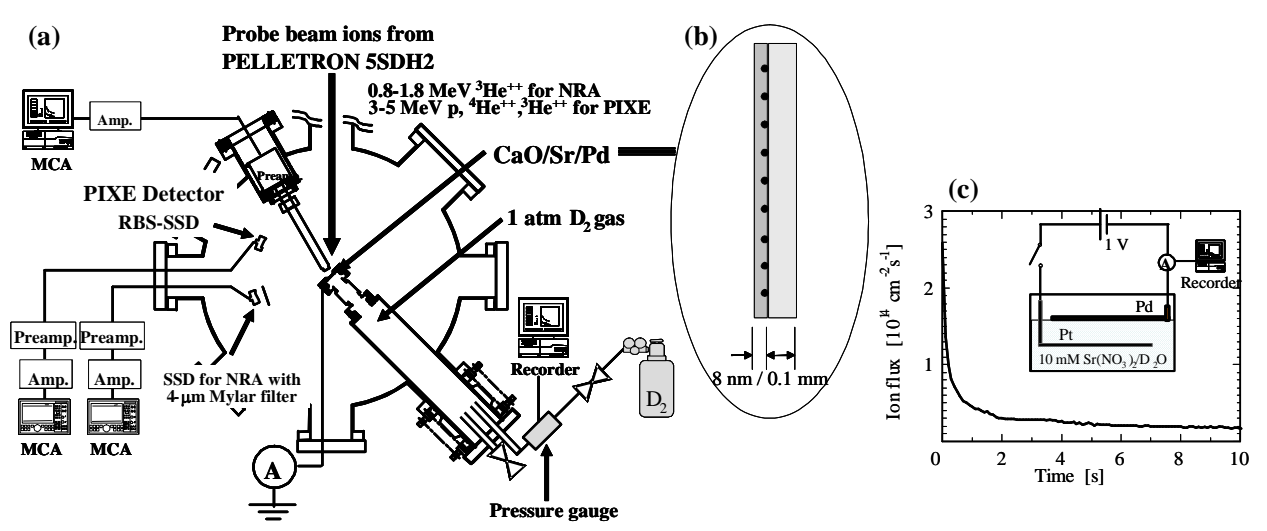

Figure 1. (a) Schematic of deuterium permeation-in situ accelerator analyses system, (b) the $\mathrm{CaO} / \mathrm{Sr} / \mathrm{PdD}_{\mathrm{x}}$ sample structure, and (c) electrochemical method used for Sr deposition onto the Pd sample.

The multilayered $\mathrm{Pd}$ samples were prepared as follows. The $\mathrm{Pd}$ sheets of $33 \times 33 \times 0.1 \mathrm{~mm}^{3}$ were annealed for 3 hours at $570 \mathrm{~K}$ after 1 -second immersion in aqua regia/ $\mathrm{D}_{2} \mathrm{O}$. Sr atoms were then deposited on one side of the Pd surface using a method similar to electroplating: As shown in Figure 1(c), the Pd sheet was carefully placed on the surface of the $10 \mathrm{mM} \mathrm{Sr}\left(\mathrm{NO}_{3}\right)_{2} / \mathrm{D}_{2} \mathrm{O}$ solution, so that only one side of the sheet was in contact with the solution. A bias voltage of $1 \mathrm{~V}$ was applied between the Pd cathode and a $\mathrm{Pt}$ anode wire immersed in the solution. The ion flow onto the $\mathrm{Pd}$ cathode was appreciable only at the beginning of the electroplating process, as can be seen in the graph, implying a saturation of the surface with contamination layer containing $\mathrm{Sr}$. Neither further increase in the processing time nor multiple immersions and biasing resulted in any increase in the areal density of Sr deposited.

Next, a CaO layer was deposited on the Sr/Pd surface by RF sputtering for 5 - $20 \mathrm{~min}$. The thickness of the $\mathrm{CaO}$ layer was deduced from variation of the XPS spectral intensities. As shown in Figure 2(a), the peak intensities of $\mathrm{Pd}-3 \mathrm{~d}$ and $\mathrm{Ca}-2 \mathrm{p}$ photoelectrons vary
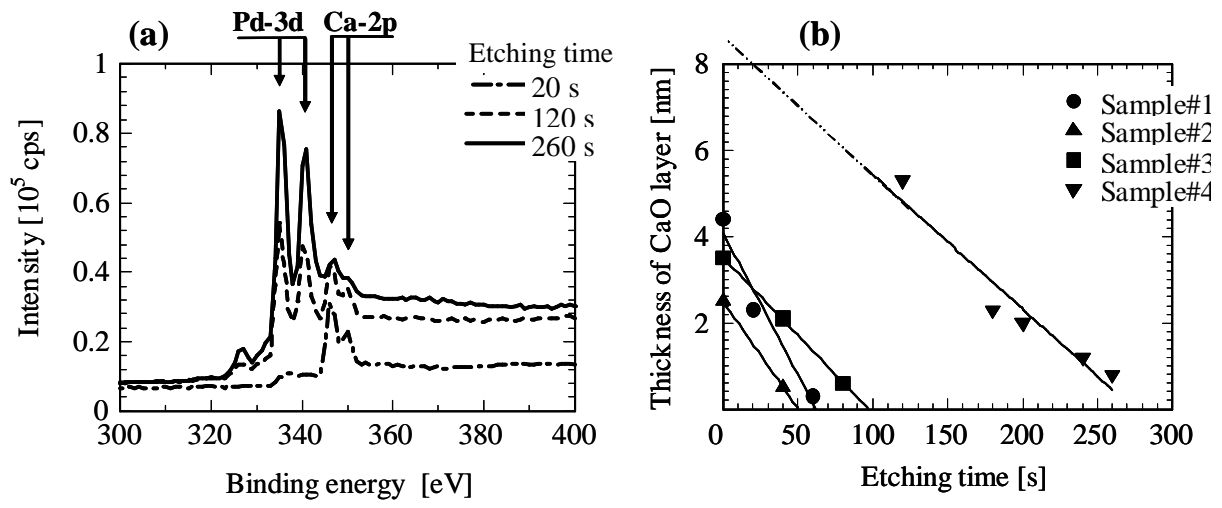

Figure 2. (a) XPS analysis of $\mathrm{CaO} / \mathrm{Pd}$ samples, and (b) determination of the $\mathrm{CaO}$ layer thickness from the XPS peak intensities. 
with surface etching time. The $\mathrm{CaO}$ layer thickness $x$ is calculated from the intensity ratio of these peaks, $Y_{\mathrm{Ca}-2 \mathrm{p}} / Y_{\mathrm{Pd}-3 \mathrm{~d}}$, using the following equation;

$$
\exp \left(\frac{x}{\lambda_{\text {CaO-Pd }}}\right) \cdot\left\{1-\exp \left(-\frac{x}{\lambda_{\text {CaO-Ca }}}\right)\right\}=\frac{n_{\text {Pd }} \cdot \sigma_{\text {Pd }} \cdot \lambda_{\text {Pd-Pd }} \cdot Y_{\text {Ca- } 2 \mathrm{p}}}{n_{\text {Ca }} \cdot \sigma_{\text {Ca }} \cdot \lambda_{\text {CaO-Ca }} \cdot Y_{\text {Pd-3d }}}
$$

where $n_{\mathrm{Ca}}, n_{\mathrm{Pd}}, \sigma_{\mathrm{Ca}}, \sigma_{\mathrm{Pd}}, \lambda_{\mathrm{CaO}-\mathrm{Ca}}, \lambda_{\mathrm{Pd}-\mathrm{Pd}}$ and $\lambda_{\mathrm{CaO}-\mathrm{Pd}}$ are the atomic density of $\mathrm{Ca}$ and Pd, the differential photoelectron emission cross section for $\mathrm{Ca}-2 \mathrm{p}$ and $\mathrm{Pd}-3 \mathrm{~d}$, and the mean free path [3] of $\mathrm{Ca}-2 \mathrm{p}$ photoelectrons in the $\mathrm{CaO}$ layer, that of $\mathrm{Pd}-3 \mathrm{~d}$ photoelectrons in the bulk $\mathrm{Pd}$ and that of $\mathrm{Pd}-3 \mathrm{~d}$ photoelectrons in the $\mathrm{CaO}$ layer, respectively. For samples thicker than several $\mathrm{nm}$, an extrapolation of the thickness to the null etching time is necessary to deduce the initial thickness of the $\mathrm{CaO}$ layer as shown in Figure 2(b).

\section{Deuterium Permeation}

A disc-shaped sample thus prepared with a diameter of $26 \mathrm{~mm}$ was placed in the vacuum chamber as shown in Figure 1. Its rear surface was exposed to $\mathrm{D}_{2}$ gas at a pressure of $0.1 \mathrm{MPa}$, while the front surface was faced to vacuum with an effective area of $2.5 \mathrm{~cm}^{2}$.

Variation of the $\mathrm{D}_{2}$ gas pressure after its introduction into the reservoir was monitored to give the number of deuterium atoms absorbed in and/or transmitted through the sample as shown in Figure 3(a). If the $\mathrm{CaO}$ layer acted as a barrier for deuterium permeation to allow negligible transmission into the vacuum, the sample would have become saturated with deuterium, i.e., $\mathrm{PdD}_{0.86}$, at about $100 \mathrm{~h}$.

This process of deuterium charging is described by a 1-dimensional solution of the diffusion equation for the sample with a thickness and area of $a$ and $S$, respectively. One side of the sample is opaque for deuterium, while the other faces hydrogen gas to give a boundary condition for the deuterium density; $n(a)=n_{0}$. The number of deuterium atoms absorbed in the sample, $N_{\mathrm{a}}(t)$, is given as a function of time by
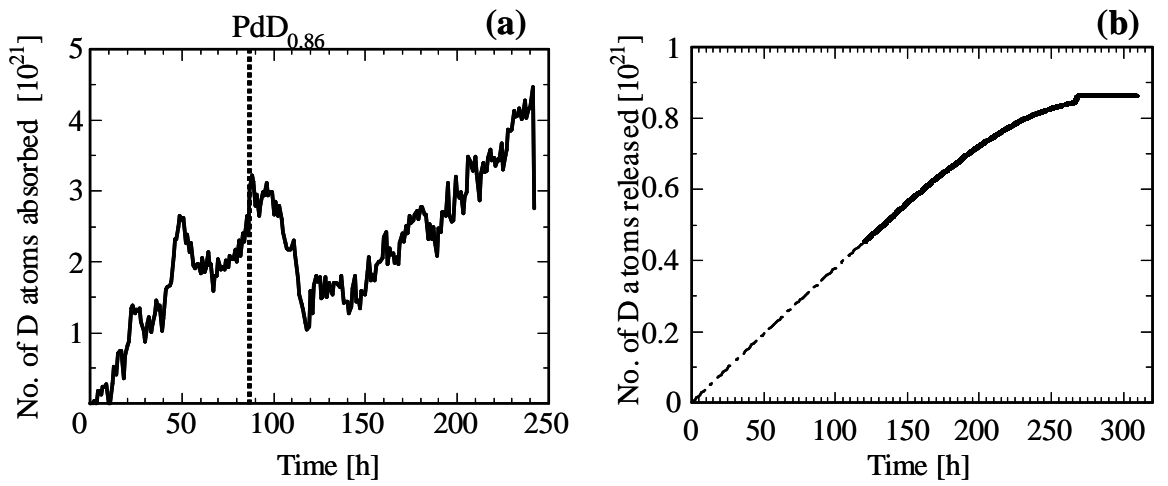

Figure 3. (a) Number of D atoms absorbed and/or transmitted during permeation, and (b) number of D atoms desorbed from the sample after finishing the permeation procedure. 


$$
N_{\mathrm{a}}(t)=a S n_{0}\left[1-\sum_{s=0}^{\infty}\left\{\frac{8}{(2 s+1)^{2} \pi^{2}} \cdot \exp \left(-\left(\frac{(2 s+1) \pi}{2 a}\right)^{2} D t\right)\right\}\right] .
$$

A characteristic time $t_{1 / 2}$ for the number of deuterium atoms absorbed to reach a half of the saturation value, i.e., $N_{\mathrm{a}}\left(t_{1 / 2}\right) / a S=N_{\mathrm{a}}(\infty) / 2 a S=2.9 \times 10^{22} \mathrm{~cm}^{-3}$, is therefore given by

$$
t_{1 / 2}=\frac{0.1967 a^{2}}{D}=6 \times 10^{1} \mathrm{~s} .
$$

The observed value is more than three orders of magnitude larger than this value. This fact implies that there is another barrier on the rear surface facing the $\mathrm{D}_{2}$ gas, possibly due to contamination. Figure 3(a) shows that the deuterium flux through the sample should have been about $2.5 \times 10^{15} \mathrm{~cm}^{-2} \mathrm{~s}^{-1}$.

After 10 days of permeation with deuterium, desorption of the $\mathrm{D}_{2}$ gas from the sample was so severe that an outgassing procedure was necessary for the sample to be introduced into the XPS vacuum system. The sample was then introduced into a vacuum chamber to measure the $\mathrm{D}_{2}$ partial pressure with a quadrupole mass spectrometer. Variation of the integrated number of deuterium atoms released during the outgassing in vacuum calculated from the partial pressure is shown as the solid line in Figure 3(b). The outgas measurement was started $120 \mathrm{~h}$ after finishing the permeation. Correction for the release into atmosphere before the measurement have been made by extrapolating the measured variation to time $t=0$. The sudden increase at about $150 \mathrm{~h}$ is due to a deliberate elevation of the temperature.

The total amount of deuterium atoms desorbed from the sample, $8.7 \times 10^{20}$, corresponds to the composition of $\mathrm{PdD}_{0.24}$. This does not always mean that the whole sample did not saturate with deuterium, when we take account of possible error in the partial pressure measurement. However, the fact that the time necessary for the release of deuterium was also of the order of $100 \mathrm{~h}$ again implies that the deuterium flow was recombination limited on both surfaces of the sample during the deuterium permeation.

\section{XPS Analysis after Deuterium Permeation}

Examples of the XPS analyses of the samples before and after deuterium permeation are compared in Figure 4(a). The samples were subjected to multiple processes of surface etching followed by analysis, giving the total etching time of $260 \mathrm{~s}$, which corresponds to the probing depth of about $8 \mathrm{~nm}$. We notice that the peak intensity of Mo- $3 \mathrm{~d}_{5 / 2}$ photoelectrons has increased drastically after the permeation in exchange for decrease in that of the $\mathrm{Sr}-3 \mathrm{~d}_{5 / 2}$.

The densities of elements found in the XPS spectra are calculated from the photoelectron yields by the conventional method using the cross sections with correction taken into account for probability of emission without scattering [3]. These are plotted in Figure $4(\mathrm{~b})$ as a function of distance from the $\mathrm{CaO} / \mathrm{Pd}$ interface calculated from the 

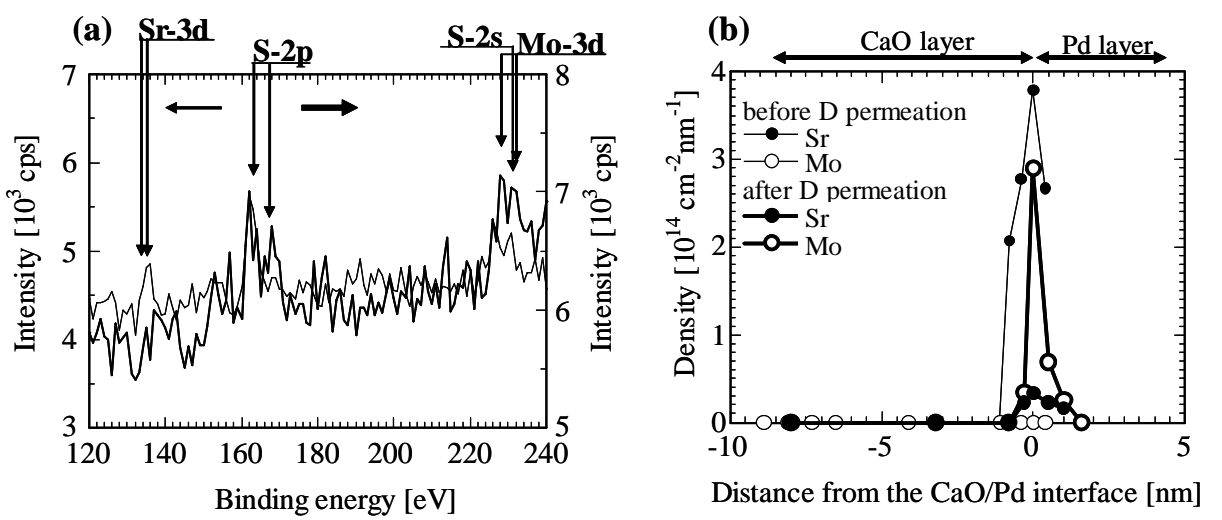

Figure 4. (a) XPS spectra for the sample before (thin line) and after (thick line) the permeation process, and (b) depth profiles of the Sr (solid circle) and Mo (open circle) densities calculated from the XPS peak intensities for the sample before (thin) and after (thick) the permeation process.

relation between the distance and the etching time shown in Figure 2(b). It is rather clearly indicated that the existence of Mo in exchange for $\mathrm{Sr}$ is recognized only near the $\mathrm{CaO} / \mathrm{Pd}$ interface after deuterium permeation. This implies occurrence of nuclear transmutation of $\mathrm{Sr}$ to Mo, i.e., $\mathrm{Sr}$ atoms with areal density of $4.2 \times 10^{14} \mathrm{~cm}^{-2}$ appear to be transformed to Mo atoms of $3.3 \times 10^{14} \mathrm{~cm}^{-2}$ by deuterium permeation. The FWHM's of the spatial distributions, $2.0-1.0 \mathrm{~nm}$, are consistent with the mean free paths of $\mathrm{Sr}-3 \mathrm{~d}_{5 / 2}$ and Mo- $3 \mathrm{~d}_{5 / 2}$ electrons of 3.8 and $3.5 \mathrm{~nm}$, respectively, in $\mathrm{CaO}$, if we take into account the statistical error, which is about $\pm 20 \%$.

The extended analytical methods are now being prepared, including the in situ accelerator analyses, will be very effective for further study. The minimum areal densities of $\mathrm{Pr}$ and Cs detectable in the upcoming PIXE analysis are estimated to be $4 \times 10^{14} \mathrm{~cm}^{-2}$ and $2 \times 10^{14} \mathrm{~cm}^{-2}$ for $100-\mathrm{p} \mu \mathrm{C} / 5-\mathrm{MeV} \alpha$-particle probing. These limiting values of the areal densities have been confirmed by preliminary analyses of an $\mathrm{Au} / \mathrm{Pd}$ sample and a $\mathrm{CaO} / \mathrm{Pd}$ sample.

\section{Summary}

Implication of nuclear transmutation of $\mathrm{Sr}$ to Mo has been obtained using a system [vacuum $\left./ \mathrm{CaO} / \mathrm{Sr} / \mathrm{PdD}_{\mathrm{x}} / \mathrm{D}_{2}\right]$, which is simpler than that of Iwamura et al., with the flow direction reversed. Reproduction of the results is necessary to confirm the transmutation.

\section{References}

1. Y. Iwamura, M. Sakano and T. Itoh; Jpn. J. Appl. Phys. 41 (2002) 4642-4650.

2. Y. Iwamura, T. Itoh, M. Sakano, S. Kuribayashi, Y. Terada, T. Ishikawa and J. Kasagi; Proc. ICCF11, 2004, Marseilles, France.

3. e.g., The Surface Science Society of Japan (ed.); X-ray Photoelectron Spectroscopy (Maruzen, Tokyo, 1998). 